\title{
Markovian Mixture Face Recognition with Discriminative Face Alignment
}

\author{
Anonymous FG2008 submission
}

\begin{abstract}
A typical automatic face recognition system is composed of three parts: face detection, face alignment and face recognition. Conventionally, these three parts are processed in a bottom-up manner: face detection is performed first, then the results are passed to face alignment, and finally to face recognition. The bottom-up approach is one extreme of vision approaches. The other extreme approach is top-down. In this paper, we proposed a stochastic mixture approach for combining bottom-up and top-down face recognition: face recognition is performed from the results of face alignment in a bottom-up way, and face alignment is performed based on the results of face recognition in a top-down way. By modeling the mixture face recognition as a stochastic process, the recognized person is decided probabilistically according to the probability distribution coming from the stochastic face recognition, and the recognition problem becomes that "who the most probable person is when the stochastic process of face recognition goes on for a long time or ideally for an infinite duration". This problem is solved with the theory of Markov chains by modeling the stochastic process of face recognition as a Markov chain. As conventional face alignment is not suitable for this mixture approach, discriminative face alignment is proposed. And we also prove that the stochastic mixture face recognition results only depend on discriminative face alignment, not on conventional face alignment. The effectiveness of our approach is shown by extensive experiments.
\end{abstract}

\section{Introduction}

A typical automatic face recognition (AFR) system is composed of three parts: face detection, face alignment and face recognition. Given images containing faces, face detection tells where the faces are, face alignment locates the key feature points of faces, and finally face recognition determines who the face is. Many algorithms have been proposed for human face recognition [5, 22]. However, they only focused on each part of the AFR system. Conventionally, these three parts are processed as follows: face detec- tion is performed first, then the detection results are passed to face alignment, and finally results of face alignment are passed to face recognition. This is a bottom-up approach, as shown in Figure 1(a). However, as we know, bottom-up is one extreme of the vision approaches. The other extreme one is the top-down approach [2].

In the bottom-up approach, each level yields data for the next level. It is a data-driven approach. It uses only class-independent information, and does not rely on classspecific knowledge. For such AFR systems, face detection and face alignment do not use the knowledge about the classes of the persons to be recognized. As it is simple and general, the bottom-up approach is at first glance appealing on neurological and computational grounds. It has influenced much classical philosophical thought and psychological theory. In order that the bottom-up approach is practical, there are two conditions that it must satisfy [2]: (a) Domain-independent processing is cheap; and (b) For each level, the input data are accurate and it yields reliable results for the next level. As face detection and face alignment are rather cheap now and they also provide reasonably reliable results, the bottom-up approach is dominant in the face recognition field. However, there are three inherent problems: (1) Class-independent face detection and face alignment may fail for some classes of persons to be recognized, although they are generally good. (2) If face detection fails to detect the face or if face alignment can not correctly locate the feature points, the face recognition will usually fail. (3) The recognition process is one-off and deterministic. Once the recognition fails, the false recognition can not be corrected later.

These problems as well as the fact that the vision process does not purely run bottom-up suggests another vision approach: the top-down approach. In the top-down approach, the higher level guides the lower level. It makes use of the class-specific knowledge. With class-specific knowledge, the top-down approach could do better for the objects where the knowledge comes from [2, 4]. However, the difficulties with the top-down approach are: (1) There may be large variations within the classes. If the variations can not be properly modelled, they will introduce unexpected errors. (2) In order to model the large variations, various models 
could be used. The problem is how to choose these models for a particular test example. (3) More efforts are needed to build model for the class-specific knowledge. With the top-down approach for the AFR system, face alignment and face detection can be built based on the classes of persons to be recognized and face recognition guides face alignment and face detection. The top-down face recognition is shown in Figure 1(b).

In order to draw on the relative merits of bottom-up and top-down approaches, a judicious mixture of them will be better [2, 3]. The mixture approach of bottom-up and topdown for face recognition is shown in Figure 1(c). We call it mixture face recognition. In this paper, we propose to incorporate class-specific knowledge to face alignment and combine it with the traditional bottom-up approach. More specifically, this paper will concentrate on combining face recognition and face alignment. Discriminative face alignment (DFA) is proposed to incorporate class-specific knowledge, where a face alignment model is trained for each person. DFA can give good results for itself and bad results for others. So, it can provide discriminative features for face recognition. With the discriminative face alignment, an stochastic mixture face recognition approach is proposed to combine the bottom-up and top-down face recognition, which is properly modeled by a Markov chain.

The rest of the paper is arranged as follows. In Section 2, we present the discriminative face alignment with active shape models. The stochastic mixture face recognition approach with Markov chain is described in Section 3. Experiments are performed in section Section 4 before conclusions are drawn in Section 5 .

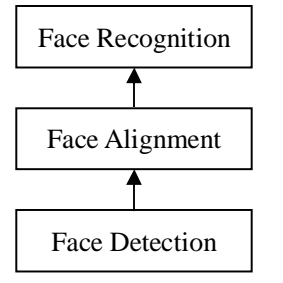

(a) Bottom-Up

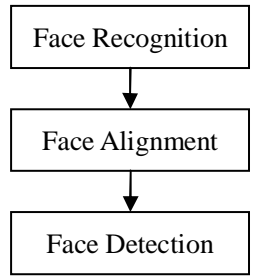

(b) Top-Down

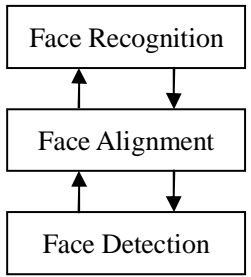

(c) Mixture
Figure 1. Face Recognition Strategies

\section{Discriminative Face Alignment}

As the top-down approach needs to incorporate classspecific knowledge to face alignment, discriminative face alignment (DFA) is proposed in this paper. It builds a face alignment model for each person to be recognized. This is different from conventional face alignment, which concentrates on general purpose face alignment (GPFA). GPFA builds the model from faces of many persons other than the persons to be recognized in order to cover the variance of all the faces. So it attains the ability of generalization at the cost of specialization. This is to serve for the bottomup approach. Moreover, GPFA doesn't consider its higherlevel tasks. However, the requirements of different tasks may be different, for example, face recognition needs discriminative features whereas face animation requires accurate positions of key points. So it would be better to consider the higher-level task for effective face alignment. As face recognition needs discriminative features, it would be better that face alignment could also give discriminative features. However, the goals of GPFA used in bottom-up approaches is accurate localization. Therefore, the performance of GPFA is not directly related to the performance of the face recognition system. On the contrary, DFA can provide accurate localization to extract good features to recognize the person on which its model is built. On the other hand, if a being-recognized person is not the person with the discriminative alignment model, the discriminative alignment model will give bad localization so as to extract bad features to prevent the being-recognized person from being recognized as the person with the discriminative alignment model. So, DFA can provide discriminative features for face recognition, which makes it better than GPFA.

\subsection{Active Shape Models}

Active Shape Models (ASM)[8] and Active Appearance Models (AAM) 6, 7] are most popular face alignment methods. ASM uses the local appearance model, which represents the local statistics around each landmark to efficiently find the target landmarks. The solution space is constrained by the properly trained global shape model. AAM combines constraints on both the shape and texture. The result shape is extracted by minimizing the texture reconstruction error. According to the different optimization criteria, ASM performs more accurately in shape localization while AAM gives a better match to image texture. In this paper, ASM is used for DFA. However, similar idea can be also applied for AAM.

ASM is composed of two parts: the shape subspace model and the search procedure. The shape subspace model is a statistical model for the tangent shape space and the search procedure is to use the local appearance models to locate the target shapes in the image. Some efforts concentrate on the search procedure [20, 15, 14, 17, 19, 9], while others focus on the subspace model [13, 10, 21]. However, all of these methods only concentrate on GPFA, called GPASM in this paper.

To train the ASM shape model, the shapes should first be annotated in the image domain. Then, these shapes are aligned into those in the tangent shape space with the Procrustes Analysis. The ASM shape model is represented by applying principle component analysis (PCA), it can be 
written as:

$$
S=\bar{S}+\Phi_{t} s
$$

where $\bar{S}$ is the mean tangent shape vector, $\Phi_{t}=\left\{\phi_{1}\left|\phi_{2}\right| \cdots \mid \phi_{t}\right\}$, which is a submatrix of $\Phi$ (the eigenvector matrix of the covariance matrix), contains the principle eigenvectors corresponding to the largest eigenvalues, and $s$ is a vector of shape parameters. for a given shape, its shape parameter is given by

$$
s=\Phi_{t}^{T}(S-\bar{S})
$$

\subsection{Discriminative Active Shape Model}

To build a discriminative active shape model, called DASM, for each person, the straightforward way is to collect some samples for each person and train the ASM model with these samples. For an AFR system, if images of each person are labelled during enrollment or registration, the DASM model could be built directly from these samples. One problem is that there should be enough variation of each person, otherwise the discriminative alignment model can not generalize well to other faces of the same person. Labelling some images is possible for each person, for example, during enrollment, images can be manually or semiautomatically labelled with the help of constrained search [7] or GP-ASM. And face variation could also be acquired for each person, for example, in the BANCA database [1], each person are recorded 5 images with face variation by speaking some words. Gross et al. [12] proposed a person specific face alignment, which is technically similar to D-ASM. However, person specific model is assumed to be built for applications where the identity of the face is known, such as interactive user interface, and it is used to improve the face alignment accuracy. It does not provide discriminative features for face recognition.

\subsection{Discriminative Features from D-ASM}

As D-ASM is able to give good alignment for itself and bad alignment for others, it can provide discriminative features for face recognition, i.e. positions of key feature points. There are small errors of key feature points for good alignment and larger errors for bad alignment. After alignment is performed, key feature points are used to extract the image patch for recognition. As D-ASM can provide accurate alignment of itself and bad alignment for others, the key feature points are discriminative for different persons.

\section{Face Recognition with Markov Chain}

In this section, the stochastic mixture face recognition is first be introduced. Then, the theory of Markov chains is presented. Finally, the mixture face recognition is modeled with a Markov chain and the recognition problem is solved with the basic limit theorem of Markov chains, which also prove that the recognition is only dependent on DFA, not GPFA.

\subsection{Stochastic Mixture Face Recognition}

However, the major problem with DFA is how to decide which model to use, which is one of the difficulties of the top-down approach as discussed in Section 1. To deal with this problem, an stochastic mixture approach is proposed to combine DFA and face recognition. The idea is shown in Figure 2. The whole recognition process works in an iterative way: face recognition is performed from the results of DFA in a bottom-up way; then, appropriate DFA models are chosen based on the results of face recognition to further improve face alignment in a top-down way; and face recognition is further improved with the improved face alignment, and the process continues in the same way. Furthermore, the mixture face recognition is performed probabilistically. It can be viewed as a stochastic process, as illustrated in Figure 3:

- For the first-round or initial recognition, GPFA is applied for face alignment. With the initial recognition result, the first-round recognized person $i_{0}$ is randomly decided according to an initial recognition probability distribution which comes from the initial recognition. This is different from the traditional deterministic recognition, in which the recognized person is chosen with the highest recognition confidence. The problem with the deterministic recognition is that once the initial recognition is wrong, there is no way to correct it. However, with the probabilistic recognition, the false initial recognition can be corrected later.

- For the second-round recognition, face alignment is performed with the discriminative face alignment model of person $i_{0}$. With the alignment result, the second-round face recognition is applied. Similar to the first-round recognition, the second-round recognized person $i_{1}$ is chosen according to the recognition probability distribution which comes from the secondround face recognition. And the recognition process goes on and on in the same way.

Now, the recognition problem becomes that who the most probable person is when the stochastic recognition process goes on for a long time or ideally for an infinite duration. This problem can be solved with the theory of Markov chains.

\subsection{Markov Chains}

"A discrete time Markov chain is a Markov process whose state space is a finite or countable set, and whose (time) index set is $T=(0,1,2, \ldots)$." “A Markov process $\left\{X_{t}\right\}$ is a stochastic process with the property that, given 


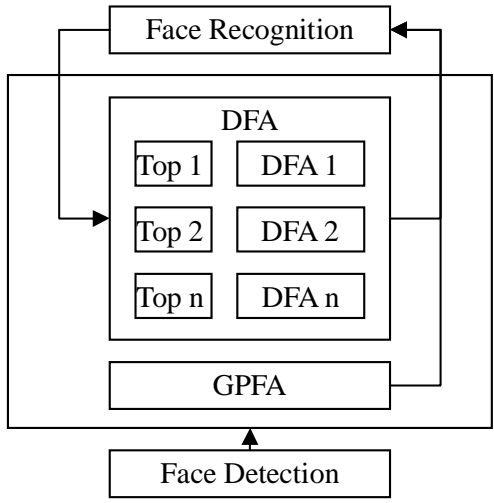

Figure 2. Mixture Face Recognition

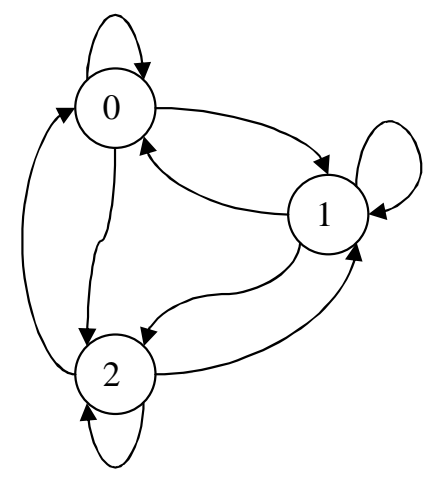

Figure 3. Stochastic Face Recognition with Markov Chain

the value of $X_{t}$, the values of $X_{s}$ for $s>t$ are not influenced by the values of $X_{u}$ for $u<t$. [16]" In formal terms, the Markov property for a discrete time Markov chain is that:

$$
\begin{gathered}
\operatorname{Pr}\left\{X_{n+1}=i_{n+1} \mid X_{n}=i_{n}, X_{n-1}=i_{n-1}, \ldots, X_{0}=i_{0}\right\} \\
=\operatorname{Pr}\left\{X_{n+1}=i_{n+1} \mid X_{n}=i_{n}\right\}
\end{gathered}
$$

where $\operatorname{Pr}\{\cdot\}$ denotes the probability function.

Given that the chain is in state $i$ at time $n$, i.e. $X_{n}=i$, the probability of the chain jumping to state $j$ at time $n+1$, i.e. $X_{n+1}=j$, is called one-step transition probability and denoted by $P_{i j}^{n, n+1}$. That is,

$$
P_{i j}^{n, n+1}=\operatorname{Pr}\left\{X_{n+1}=i_{j} \mid X_{n}=i\right\}
$$

If $P_{i j}^{n, n+1}$ is independent of the variable $n$, we say that the Markov chain has stationary transition probabilities, i.e. $P_{i j}^{n, n+1}=P_{i j}$. In this case, the matrix $\mathbf{P}=\left\{P_{i j}\right\}$ is called the transition probability matrix.

To specify a discrete time Markov chain with stationary transition probabilities, three kinds of parameters are needed:

- State space $\mathbb{S}$. $\mathbb{S}$ is a finite or countable set of states that the random variables $X_{i}$ may take on. For a finite set of states, the state space can be denoted as $\mathbb{S}=$ $\{1,2, \ldots, N\}$.

- Initial distribution $\pi_{0}$. This is the probability distribution of the Markov chain at time 0. For each state $i \in \mathbb{S}$, we denote by $\pi_{0}(i)$ the probability $\operatorname{Pr}\left\{X_{0}=i\right\}$ that the Markov chain starts out in state $i . \pi_{0}(i)$ satisfies the following conditions

$$
\begin{gathered}
\pi_{0}(i) \geq 0 \quad(i \in \mathbb{S}) \\
\sum_{i \in \mathbb{S}} \pi_{0}(i)=1
\end{gathered}
$$

- Transition probability matrix $\mathbf{P}=\left\{P_{i j}\right\} . P_{i j}$ is the probability of transition from state $i$ to state $j$. It satisfies the following conditions

$$
\begin{gathered}
P_{i j} \geq 0 \quad(i, j \in \mathbb{S}) \\
\sum_{j \in \mathbb{S}} P_{i j}=1 \quad(i \in \mathbb{S})
\end{gathered}
$$

In the rest of this paper, "Markov chain" represents "discrete time Markov chain with stationary transition probabilities".

\subsection{Markov Chain for Mixture Face Recognition}

The stochastic mixture face recognition process can be modeled with a Markov chain. As introduced in Section 3.2, three kinds of parameters are needed to be specified:

- State space $\mathbb{S}$. The states are the persons to be recognized. Suppose that there are $N$ persons to be recognized. So, the state space can be denoted as $\mathbb{S}=$ $\{1,2, \ldots, N\}$.

- Initial distribution $\pi_{0}$. This is the first-round or initial recognition probability distribution coming from the initial face recognition. GPFA is used for face alignment.

Assume that the face recognition algorithm produces recognition distance $d_{r}^{i}$ for person $i$. Then, a weight $w_{i}$ is associated with each person, which is defined as:

$$
w_{i}=\exp \left(-\frac{d_{r}^{i}}{\sigma^{2}}\right)
$$

Then, the initial probability for person $i$ is

$$
\pi_{0}(i)=\frac{w_{i}}{\sum_{i=0}^{N} w_{i}}
$$

- Transition probability matrix $\mathbf{P}=\left\{P_{i j}\right\} . P_{i j}$ is the probability with which person $j$ will be recognized in the next round when person $i$ is recognized in current round. DFA is used with the model of person $i$. The combined recognition distance from Equation (??) is 
used. A weight $w_{i j}$ is defined between person $i$ and $j$ as follows:

$$
w_{i j}=\exp \left(-\frac{d_{r}^{i j}}{\sigma^{2}}\right)
$$

Then, the transition probability from person $i$ to person $j$ is defined as:

$$
P_{i j}=\frac{w_{i j}}{\sum_{i=0}^{N} w_{i j}}
$$

With this modeling, the face recognition problem, i.e. who the most probable person is when the stochastic recognition process goes on for a long time or ideally for an infinite duration, can be solved by the limiting distribution of Markov chains. The limiting distribution $\pi$ means that after the process has been in operation for a long duration the probability of finding the process in state $i$ is $\pi(i)$. So, the most probable person is the person with the highest $\pi(i)$.

Because all the elements are strictly positive, the transition probability matrix for mixture face recognition is regular [16]. According to the basic limit theorem of Markov chains, a Markov chain with a regular transition probability matrix has a limiting distribution $\pi$ which is the unique nonnegative solution of the following equations:

$$
\begin{array}{r}
\pi=\pi \mathbf{P} \\
\sum_{i \in \mathbb{S}} \pi(i)=1
\end{array}
$$

Equations (13) and (14) shows that the limiting distribution is only dependent on the transition matrix $\mathbf{P}$, not on the initial distribution $\pi_{0}$. In other words, it proves that the recognition only depends on the DFA (which is used to generating $\mathbf{P}$ ), not on GPFA (which is used to generating $\pi_{0}$ ).

\section{Experiments}

In this section, we perform experiments on the BANCA face database [1]. First, experiments is experiments is conducted to evaluate the discriminative features from D-ASM. Then, the recognition is evaluated.

The CSU Face Identification Evaluation System [11] is utilized to test the performance of the stochastic mixture face recognition.

Face detection is performed with an AdaBoost face detector [18]. For images with no detected face or more than two detected faces, we manually give the the face detection or manually choose the correctly detected face. After face alignment, the images are registered using eye coordinates and cropped with an elliptical mask to exclude nonface area from the image. After this, the grey histogram over the non-masked area is equalized.

\subsection{Discriminative Features from Discriminative Face Alignment}

This subsection will validate the statements in Section 2.3 that discriminative face alignment can provide discriminative features. The experiments are performed on the BANCA database. We manually labelled 5 images of each of the 52 persons in session 1 and these images are used to train D-ASM. And GP-ASM is trained on the labelled images in session 1 from the other group, i.e. from G1 and G2 alternatively. The testing images are from session 2, each person with 2 images whose faces are manually labelled. So there are totally 104 testing images. The results are evaluated by the average reconstruction error (RecErr) and the average point-to-point errors of all the feature points (AllErr), the key feature points (KeyErr) (including eye centers, nose tip and mouth center) and eye centers (EyeErr) . To get the reconstruction error, the texture PCA model is built from another 200 labelled faces. Four kinds of experiments are conducted: (1) GP-ASM-A: GP-ASM is used to align all the testing images; (2) D-ASM-O: D-ASM is used to align all the testing images of other persons. (3) D-ASMS: D-ASM is used to align only the testing images of itself. Results are shown in Table 1. Some examples are shown in Figure 4. Figure 4(a)-(c) is for GP-ASM-A; Figure 4(d)-(f) is for D-ASM-O; Figure 4(g)-(i) is for D-ASM-S. These results show that D-ASM-S gives more accurate results than GP-ASM, and it can give significantly better results than DASM-O. This clearly shows that D-ASM can provide discriminative features.

\begin{tabular}{|c|c|c|c|c|}
\hline & AllErr & KeyErr & EyeErr & RecErr \\
\hline GP-ASM-A & 4.88 & 3.33 & 3.23 & 15.18 \\
\hline D-ASM-O & 9.75 & 6.93 & 6.67 & 36.77 \\
\hline D-ASM-S & 3.05 & 2.20 & 2.10 & 13.42 \\
\hline \multicolumn{4}{|c}{ Table 1. Results of GP-ASM and D-ASM }
\end{tabular}

\subsection{Mixture Face Recognition on BANCA}

The BANCA database contains 52 subjects (26 males and 26 females). Each subject participated in 12 recording sessions in different conditions and with different cameras. Session 1-4 contain data under controlled conditions while sessions 5-8 and 9-12 contain degraded and adverse scenarios respectively. To minimize the impact of illumination and image quality, we choose to use session 1-4.

For BANCA, we manually labeled 87 landmarks for session 1 , i.e. totally $260(=52 * 5)$ faces. Session 2 - 4 client attack faces are used for testing, totally $780(=52 * 15)$ faces. So, there are 260 faces as gallery, and 780 faces as probe.

We manually labelled the training set of MC configuration for each person with five images in session 1 . DASM is trained on five images for each person; and GP- 


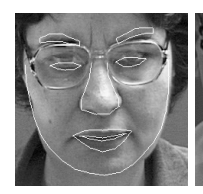

(a)

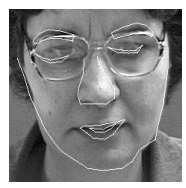

(d)

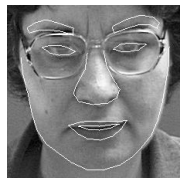

(g)

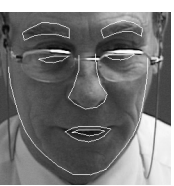

(b)

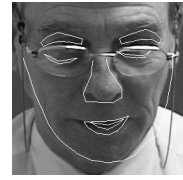

(e)

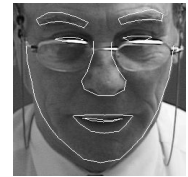

(h)

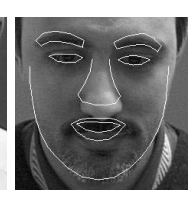

(c)

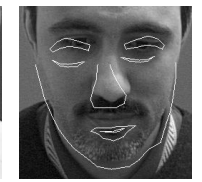

(f)

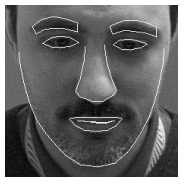

(i)
Figure 4. Face Alignment Examples of GP-ASM and D-ASM

ASM is trained on the labelled images in session 1 from the other group, i.e. from G1 and G2 alternatively. Results are shown in Table ??, where PCA-M and LDA-M stands for the stochastic mixture recognition with PCA and LDA. From the results, we can find that the stochastic mixture face recognition can consistently and significantly improve the performance.

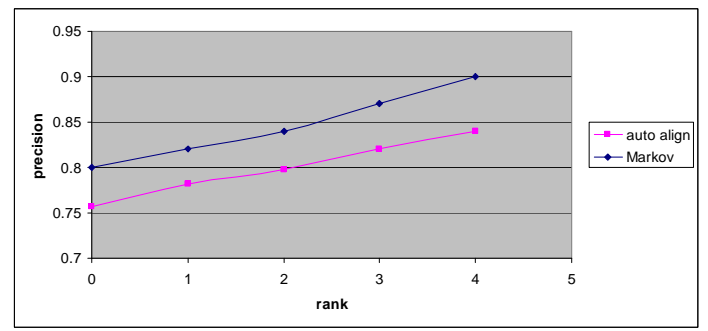

Figure 5. Face Recognition Results with PCA

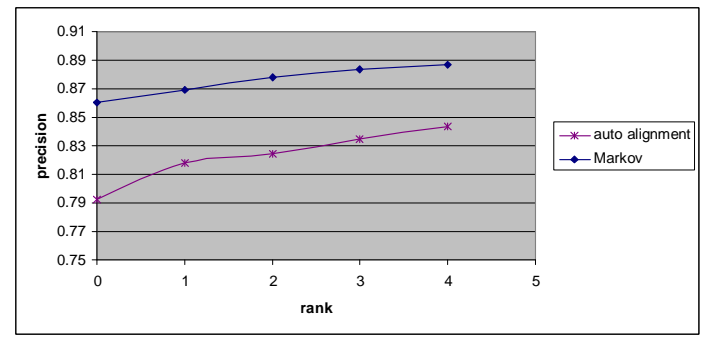

Figure 6. Face Recognition Results with LDA

\section{Conclusions}

Conventional face recognition is only a bottom-up approach. This paper proposed to use the top-down approach and combine it with the bottom-up approach. In particular, a stochastic mixture approach is proposed for combining face alignment and face recognition. The recognition process works as a stochastic process, and it is properly modeled by a Markov chain, and the recognition problem is solved with the basic limit theorem of Markov chains. Discriminative face alignment is also proposed to incorporate classspecific knowledge, and it can provide discriminative features for better face recognition. Proof is done to show that the face recognition results are dependent only on discriminative face alignment, not on conventional face alignment. Experiments demonstrated that the mixture face recognition algorithms can consistently and significantly improve the face recognition performance. Future work includes improving other face recognition algorithms with the stochastic mixture face recognition and incorporating face detection in the whole mixture face recognition framework.

\section{References}

[1] E. Bailly-Bailliére, S. Bengio, F. Bimbot, M. Hamouz, J. Kittler, J. Mariéthoz, J. Matas, K. Messer, V. Popovici, F. Porée, B. Ruiz, and J.-P. Thiran. The BANCA database and evaluation protocol. In 4th International Conference on Audioand Video-Based Biometric Person Authentication, Surrey, Berlin, 2003. Springer-Verlag. 3, 5]

[2] D. H. Ballard and C. M. Brown. Computer Vision, chapter 10, pages 340-348. Prentice-Hall, 1982. 1, 2

[3] E. Borenstein, E. Sharon, and S. Ullman. Combining topdown and bottom-up segmentation. volume 04, 2004. 2

[4] E. Borenstein and S. Ullman. Class-specifc, top-down segmentation. In ECCV, pages 109-122, Copenhagen, Denmark, May 28-31 2002. 1

[5] R. Chellappa, C. Wilson, and S. Sirohey. Human and machine recognition of faces: A survey. Proceedings of the IEEE, 83:705-740, 1995. 1

[6] T. F. Cootes, G. J. Edwards, and C. J. Taylor. Active appearance models. In ECCV98, volume 2, pages 484-498, 1998. 2

[7] T. F. Cootes and C. J. Taylor. Constrained active appearance models. In Proceedings of IEEE International Conference on Computer Vision, pages 748-754, Vancouver, Canada, July 2001. 2] 3 .

[8] T. F. Cootes, C. J. Taylor, D. H. Cooper, and J. Graham. Active shape models: Their training and application. CVGIP: Image Understanding, 61:38-59, 1995. 2]

[9] C. D. and C. T.F. A comparison of shape constrained facial feature detectors. In Proceedings of IEEE International Conference on Automatic Face and Gesture Recognition, pages 375-380, May 2004. 2

[10] R. H. Davies, T. F. Cootes, and C. J. Taylor. A minimum description length approach to statistical shape modelling. 
[11] B. D.S., B. J.R., T. M., and D. B.A. The csu face identification evaluation system: Its purpose, features and structure. In Third International Conference on Computer Vision Systems, pages $304-311,2003$. 5

[12] R. Gross, I. Matthews, and S. Baker. Generic vs. person specific active appearance models. In British Machine Vision Conference, September 2004. 3

[13] A. Hill and C. J. Taylor. A framework for automatic landmark identification using a new method of nonrigid correspondence. IEEE Transactions on Pattern Analysis and Machine Intelligence, 22(3). 2

[14] C. Liu, H.-Y. Shum, and C. Zhang. Hierarchical shape modeling for automatic face localization. In Proceedings of the European Conference on Computer Vision, number II, pages 687-703, Copenhagen, Denmark, May 2002. 2

[15] M. Rogers and J. Graham. Robust active shape model search. In Proceedings of the European Conference on Computer Vision, number IV, pages 517-530, Copenhagen, Denmark, May 2002. 2

[16] H. M. Taylor and S. Karlin. An Introduction to Stochastic Modeling. Academic Press, 3 edition, 1998. 4, 5

[17] B. van Ginneken, A. F. Frangi, J. J. Staal, B. M. ter Haar Romeny, and M. A. Viergever. Active shape model segmentation with optimal features. IEEE Transactions on Medical Imaging, 21(8):924-933, August 2002. 2

[18] P. Viola and M. Jones. Robust real time object detection. In IEEE ICCV Workshop on Statistical and Computational Theories of Vision, Vancouver, Canada, July 13 2001. 5

[19] S. Yan, M. Li, H. Zhang, and Q. Cheng. Ranking prior likelihood distributions for bayesian shape localization framework. In Proceedings of IEEE International Conference on Computer Vision, volume 1, pages 51 - 58, Nice, France, October 2003. 2

[20] S. C. Yan, C. Liu, S. Z. Li, L. Zhu, H. J. Zhang, H. Shum, and Q. Cheng. Texture-constrained active shape models. In Proceedings of the First International Workshop on GenerativeModel-Based Vision (with ECCV), Copenhagen, Denmark, May 2002. 2

[21] M. Zhao and T.-S. Chua. Face alignment with unified subspace optimization for active statistical models. In The 7th IEEE International Conference on Automatic Face and Gesture Recognition, pages 67-72, Southampton, UK, April 2006. 2]

[22] W. Zhao, R. Chellappa, A. Rosenfeld, and P. Phillips. Face recognition: A literature survey. ACM Computing Surveys, 35(4):399-458, December 2003. 1 